\title{
Temperature Scaling in Flat Space Cosmology in Comparison to Standard Cosmology
}

\author{
Eugene Terry Tatum ${ }^{1}$, U. V. S. Seshavatharam ${ }^{2}$ \\ ${ }^{1} 760$ Campbell Ln. Ste., Bowling Green, KY, USA \\ ${ }^{2}$ Honorary Faculty, I-SERVE, Hyderabad, India \\ Email: ett@twc.com, seshavatharam.uvs@gmail.com
}

How to cite this paper: Tatum, E.T. and Seshavatharam, U.V.S. (2018) Temperature Scaling in Flat Space Cosmology in Comparison to Standard Cosmology. Journal of Modern Physics, 9, 1404-1414. https://doi.org/10.4236/jmp.2018.97085

Received: May 2, 2018

Accepted: June 5, 2018

Published: June 8, 2018

Copyright ( 92018 by authors and Scientific Research Publishing Inc. This work is licensed under the Creative Commons Attribution International License (CC BY 4.0).

http://creativecommons.org/licenses/by/4.0/

(c) (i) Open Access

\begin{abstract}
Flat Space Cosmology (FSC) is a mathematical model of universal expansion which has proven to be remarkably accurate in comparison to observations. Temperature scaling is redefined in this paper in terms of a new "Universal Temperature" $T_{u}$ scale according to $T_{u}=T^{2}$, where $T^{2}$ is in $\mathrm{K}^{2}$. This rescaling puts FSC cosmic temperature, time, total matter mass, and Hubble radius on the same scale, covering roughly 60.63 logs of 10 from the Planck scale to the present scale. This paper focuses on the relatively subtle temperature curve differences between the FSC model and standard cosmology. These changes become more pronounced in the early universe. Recent observational studies of the early universe, particularly with respect to the "cosmic dawn" epoch, the first stars and first galaxies, have surprised standard model proponents as to how soon these events have occurred following the Big Bang. This paper suggests that, because the FSC model temperature/time curve is lower at each stage of cosmic time, FSC may actually be a better fit for the timing of these events.
\end{abstract}

\section{Keywords}

Cosmology Theory, General Relativity, CMB Temperature, Cosmic Flatness, Black Holes, Universal Temperature

\section{Introduction}

Flat Space Cosmology (FSC) was developed as a heuristic of the Hawking-Penrose idea of treating the universe expanding from a singularity state as being equivalent to a time-reversed gigantic black hole (i.e., one which smoothly expands from a singularity as opposed to smoothly collapsing to a singularity). Penrose had started the development with his initial paper on gravitational collapse and 
space-time singularities [1]. Hawking's doctoral thesis advanced the idea by proving the validity, within general relativity, of the black hole time-reversal idea [2]. And finally, FSC has shown very clearly that the appropriate scaling black hole equations are remarkably accurate in modeling our expanding universe [3] [4] [5].

Recently, we have integrated the FSC equations into the Friedmann equations incorporating a cosmological term for vacuum energy (dark energy). One of the results of integrating FSC into the Friedmann equations is that the following relation holds true in FSC:

$$
\frac{3 H^{2} c^{2}}{8 \pi G} \cong \frac{\Lambda c^{4}}{8 \pi G}
$$

This equation is a consequence of modeling a spatially flat universe from its inception. It should be remembered that, to date, there is no observational proof that our expanding universe has been anything other than spatially flat. At least as far back in time as the CMB radiation release event ("recombination epoch"), our universe appears to be spatially flat [6]. The extreme flatness of our universe presents a "cosmic flatness problem," first elucidated by physicist Robert Dicke in the late 1960's [7] [8]. In fact, theories of cosmic inflation [9] [10] [11] [12] were invented primarily to address this problem. The FSC model, on the other hand, tackles the flatness problem in an entirely different way. Since perpetual spatial flatness on a global scale (i.e., for a cosmic model as a whole) is not forbidden in a general relativity model of a finite but unbounded expanding universe, FSC starts with the (implied) assumption that the curvature term $k$ in the FSC Friedmann equations is perpetually zero. Thus, Equation (1) in this paper is a direct consequence of this assumption.

In so many words, Equation (1) says that, in the FSC general relativity model, the magnitude of the positive (i.e., gravitationally attracting) matter energy density is always equal in absolute magnitude to the negative (dark) energy density. This is fully understandable in terms of general relativity because a globally spatially flat universe, by definition, must have a net zero energy density. If the case were otherwise, the greater energy density term would dictate a global spatial curvature, either positive or negative, depending upon the sign of the dominating term.

In standard cosmology, there is tension between the observations of extreme global spatial flatness and the popular assumption of dark energy dominance. This makes no sense. It can only be one or the other, but not both at the same time. It should be remembered that cosmic acceleration is still not proven, despite the fact that dark energy is now known to exist [13] [14] [15]. Realistic $R_{h}=$ ct universe models, such as FSC, are now the chief competition for standard cosmology [16] [17] [18] [19] [20]. These recent statistical studies of the Supernova Cosmology Project data clearly demonstrate that cosmic acceleration is not yet proven to the exclusion of cosmic coasting models like $R_{h}=c t$. FSC incorporates dark energy in perpetual perfect balance with the effect of attractive gravity 
on a global scale. FSC accomplishes this by

$$
\Lambda \cong \frac{3 H^{2}}{c^{2}}
$$

This is the only way that Equation (1) holds true. Since $H_{t}$ is a changing parameter over the great span of cosmic time, this makes FSC a dynamic dark energy model of the wCDM type, with the equation of state in FSC always defined as $w=-1$. This is in keeping with the quantum field theory stipulation that the zero-state vacuum energy density must always be equal in magnitude to the vacuum pressure (i.e., $\rho=p$ ).

Given this background, the current basic five assumptions of FSC are presented below.

\section{The Five Assumptions of Flat Space Cosmology}

1) The cosmic model is an ever-expanding sphere such that the cosmic horizon always translates at speed of light $c$ with respect to its geometric center at all times $t$. The observer is defined to be at this geometric center at all times $t$.

2) The cosmic radius $R_{t}$ and total mass $M_{t}$ follow the Schwarzschild formula $R_{t} \cong 2 G M_{t} / c^{2}$ at all times $t$.

3) The cosmic Hubble parameter $H_{t} \cong c / R_{t}$ at all times $t$.

4) Incorporating our cosmological scaling adaptation of Hawking's black hole temperature formula, at any radius $R_{t}$ cosmic temperature $T_{t}$ is inversely proportional to the geometric mean of cosmic total mass $M_{t}$ and the Planck mass $M_{p l} . R_{p l}$ is defined as twice the Planck length (i.e., as the Schwarzschild radius of the Planck mass black hole). With subscript $t$ for any time stage of cosmic evolution and subscript $p l$ for the Planck scale epoch, and, incorporating the Schwarzschild relationship between $M_{t}$ and $R_{t}$

$$
\begin{aligned}
& k_{B} T_{t} \cong \frac{\hbar c^{3}}{8 \pi G \sqrt{M_{t} M_{p l}}} \cong \frac{\hbar c}{4 \pi \sqrt{R_{t} R_{p l}}} \\
& \left\{\begin{array}{l}
M_{t} \cong\left(\frac{\hbar c^{3}}{8 \pi G k_{B} T_{t}}\right)^{2} \frac{1}{M_{p l}} \\
R_{t} \cong \frac{1}{R_{p l}}\left(\frac{\hbar c}{4 \pi k_{B}}\right)^{2}\left(\frac{1}{T_{t}}\right)^{2} \\
R_{t} T_{t}^{2} \cong \frac{1}{R_{p l}}\left(\frac{\hbar c}{4 \pi k_{B}}\right)^{2} \\
H_{t} \cong \frac{c}{R_{t}}
\end{array}\right\}
\end{aligned}
$$

5) Total entropy of the cosmic model follows the Bekenstein-Hawking black hole formula [21] [22].

$$
S_{t} \cong \frac{\pi R_{t}^{2}}{L_{p}^{2}}
$$


The strength of the FSC model is largely due to the consistent theme behind these assumptions (i.e., the Hawking-Penrose idea) and the use of our scaling Hawking black hole temperature formula [the top equation in relations (3)], which is more appropriate for an expanding and scaling cosmological model. By virtue of the fact that Hawking's original static black hole temperature formula incorporated elementary and fundamental constants of nature, our scaling FSC model, based upon adapting Hawking's static formula, is the first quantum cosmology model. The five basic FSC assumptions, incorporated into this scaling temperature formula, dictate the following quantum cosmology equations

$$
\begin{aligned}
R & \cong \frac{\hbar^{3 / 2} c^{7 / 2}}{32 \pi^{2} k_{B}^{2} T^{2} G^{1 / 2}}, \quad R_{0} \cong \frac{\hbar^{3 / 2} c^{7 / 2}}{32 \pi^{2} k_{B}^{2} T_{0}^{2} G^{1 / 2}} \\
H \cong \frac{32 \pi^{2} k_{B}^{2} T^{2} G^{1 / 2}}{\hbar^{3 / 2} c^{5 / 2}}, & H_{0} \cong \frac{32 \pi^{2} k_{B}^{2} T_{0}^{2} G^{1 / 2}}{\hbar^{3 / 2} c^{5 / 2}} \\
t & \cong \frac{\hbar^{3 / 2} c^{5 / 2}}{32 \pi^{2} k_{B}^{2} T^{2} G^{1 / 2}}, \quad t_{0} \cong \frac{\hbar^{3 / 2} c^{5 / 2}}{32 \pi^{2} k_{B}^{2} T_{0}^{2} G^{1 / 2}} \\
M & \cong \frac{\hbar^{3 / 2} c^{11 / 2}}{64 \pi^{2} k_{B}^{2} T^{2} G^{3 / 2}}, \quad M_{0} \cong \frac{\hbar^{3 / 2} c^{11 / 2}}{64 \pi^{2} k_{B}^{2} T_{0}^{2} G^{3 / 2}} \\
M c^{2} \cong \frac{\hbar^{3 / 2} c^{15 / 2}}{64 \pi^{2} k_{B}^{2} T^{2} G^{3 / 2}}, & M_{0} c^{2} \cong \frac{\hbar^{3 / 2} c^{15 / 2}}{64 \pi^{2} k_{B}^{2} T_{0}^{2} G^{3 / 2}}
\end{aligned}
$$

Current observational parameters are calculated in the right-hand column. The only free parameter in any of these equations is the cosmic temperature. The currently observed cosmic temperature value: $T_{0}=2.72548 \mathrm{~K}$. By incorporating the known values of $T_{0}, \hbar, G, k_{B}, L_{p}$, and $\pi$ to as many decimal places as possible, the current FSC derived Hubble parameter value is

$$
H_{0}=2.167862848658891 \times 10^{-18} \mathrm{~s}^{-1}\left(66.89325791854758 \mathrm{~km} \cdot \mathrm{s}^{-1} \cdot \mathrm{Mpc}^{-1}\right)
$$

This derived theoretical global $H_{o}$ value fits very closely with the low end range of the 2015 Planck Collaboration observational global $H_{o}$ value of $67.8 \pm$ $0.9 \mathrm{~km} \cdot \mathrm{s}^{-1} \cdot \mathrm{Mpc}^{-1}$ (68\% confidence interval). And yet, this theoretical calculation is based only upon the one free parameter: $T_{0}=2.72548 \mathrm{~K}$. This is a remarkable result!

Therefore, one can have great confidence that the following cosmological parameters incorporating the FSC derived $H_{o}$ value are also highly accurate. Although one can certainly use the FSC quantum cosmology formulae given above, recalculating each additional parameter using the known constants of nature taken out to many decimal places can be a bit cumbersome. Using the once-derived $H_{o}$ value given above can take the place of these more cumbersome calculations.

$$
t_{0} \cong \frac{1}{H_{0}}=4.612837941379141 \times 10^{17} \mathrm{~s}\left(14.61694683819266 \times 10^{9} \text { sidereal yrs }\right)
$$

(multiplying by 1 sidereal $\mathrm{yr} / 3.155814954 \times 10^{7} \mathrm{~s}$ ). 
This value is the reciprocal of the derived Hubble parameter, as one would expect for a flat space-time cosmic model in comparison to the standard inflationary model. 13.7 billion years is now consensus for the standard model.

$$
R_{0} \cong \frac{c}{H_{0}}=1.38289402480171 \times 10^{26} \mathrm{~m}\left(14.61720137583068 \times 10^{9} \text { light-yrs }\right)
$$

(multiplying by 1 Julian light-yr/9.4607304725808 $\times 10^{15} \mathrm{~m}$ ).

This current cosmic radius value correlates with current cosmic time by $R_{0}=c t_{0}$. For reasons given in the seminal FSC papers, a perpetually flat and finite space-time cosmology model has no need to incorporate a superluminal inflationary mechanism to solve the flatness and horizon problems.

$$
\begin{gathered}
V o l_{0}=\frac{4 \pi}{3}\left(\frac{c}{H_{0}}\right)^{3}=1.107784564915062 \times 10^{79} \mathrm{~m}^{3} \\
M_{0}=\frac{c^{3}}{2 G H_{0}}=9.311265291518025 \times 10^{52} \mathrm{~kg}
\end{gathered}
$$

This total mass number can be compared very favorably to a rough estimate made from astronomical observations. The visible matter consists of roughly 100 billion galaxies averaging roughly 100 billion stars each, of average star mass equal to roughly $1.4 \times 10^{30} \mathrm{~kg}$ (70 percent of solar mass), totaling to roughly 1.4 $\times 10^{52} \mathrm{~kg}$. The 2015 Planck Collaboration report indicates a universal matter ratio of approximately 5.47 parts dark matter to 1 part visible (baryonic) matter. This brings the total estimated matter in the observable universe to approximately $9.1 \times 10^{52} \mathrm{~kg}$. A recent study [23] of average mass density of intergalactic dust gives a value of approximately $10^{-30} \mathrm{~kg} \cdot \mathrm{m}^{-3}$. Since this is approximately 1 part intergalactic dust to 1000 parts galactic and perigalactic matter, intergalactic dust does not appreciably modify the estimated total observational mass of matter given above. Accordingly, this observational estimate is remarkably close to the above FSC theoretical calculation of total cosmic mass attributed to positive (i.e., gravitationally attractive) matter. By the FSC Friedmann equations, the positive matter mass-energy is equal in absolute magnitude to the negative (dark) energy at all times. This is a 50/50 percentage ratio as opposed to the approximately 30/70 ratio implied by (yet unproven) cosmic acceleration. However, without proving cosmic acceleration, standard cosmology cannot claim this $30 / 70$ ratio!

$$
\begin{gathered}
M_{0} c^{2}=\frac{c^{5}}{2 G H_{0}}=8.368547901344209 \times 10^{69} \mathrm{~J} \\
\rho_{0}=\frac{3 H_{0}^{2}}{8 \pi G}=8.405303329200976 \times 10^{-27} \mathrm{~kg} \cdot \mathrm{m}^{-3} \text { (critical mass density) }
\end{gathered}
$$

This closely approximates the observational cosmic mass density calculation of critical density.

$$
\rho_{0} c^{2}=\frac{3 H_{0}^{2} c^{2}}{8 \pi G}=7.554309895973191 \times 10^{-10} \mathrm{~J} \cdot \mathrm{m}^{-3}
$$


This closely approximates the observational cosmic mass-energy density and the observational vacuum energy density. They are equal in absolute magnitude in FSC.

Given the above background on the validity of FSC as a general relativity cosmological model, the purpose of this paper is to explain how and why the temperature curve in FSC differs slightly from that of the standard cosmology model, and to explain why this may be important in terms of observational studies of the early universe.

\section{New Temperature Scaling in Flat Space Cosmology}

Using the temperature formulae of FSC assumption 4, and incorporating the Schwarzschild formula and Hubble parameter definition of FSC assumptions 2 and 3, respectively, the following four axis log graph (Figure 1) can be created for the expanding cosmic model.

This graph shows the FSC inter-relationships of the cosmic temperature, cosmic time, total cosmic mass of gravitational matter, and the cosmic Hubble radius. Notice that, while the cosmic Kelvin scale temperature decreases by approximately 30.315 logs of 10 from the Planck scale to the scale of the current observable universe, the parameters represented on the other three axes scale by approximately $60.63 \operatorname{logs}$ of 10 . This is in keeping with the following formulae

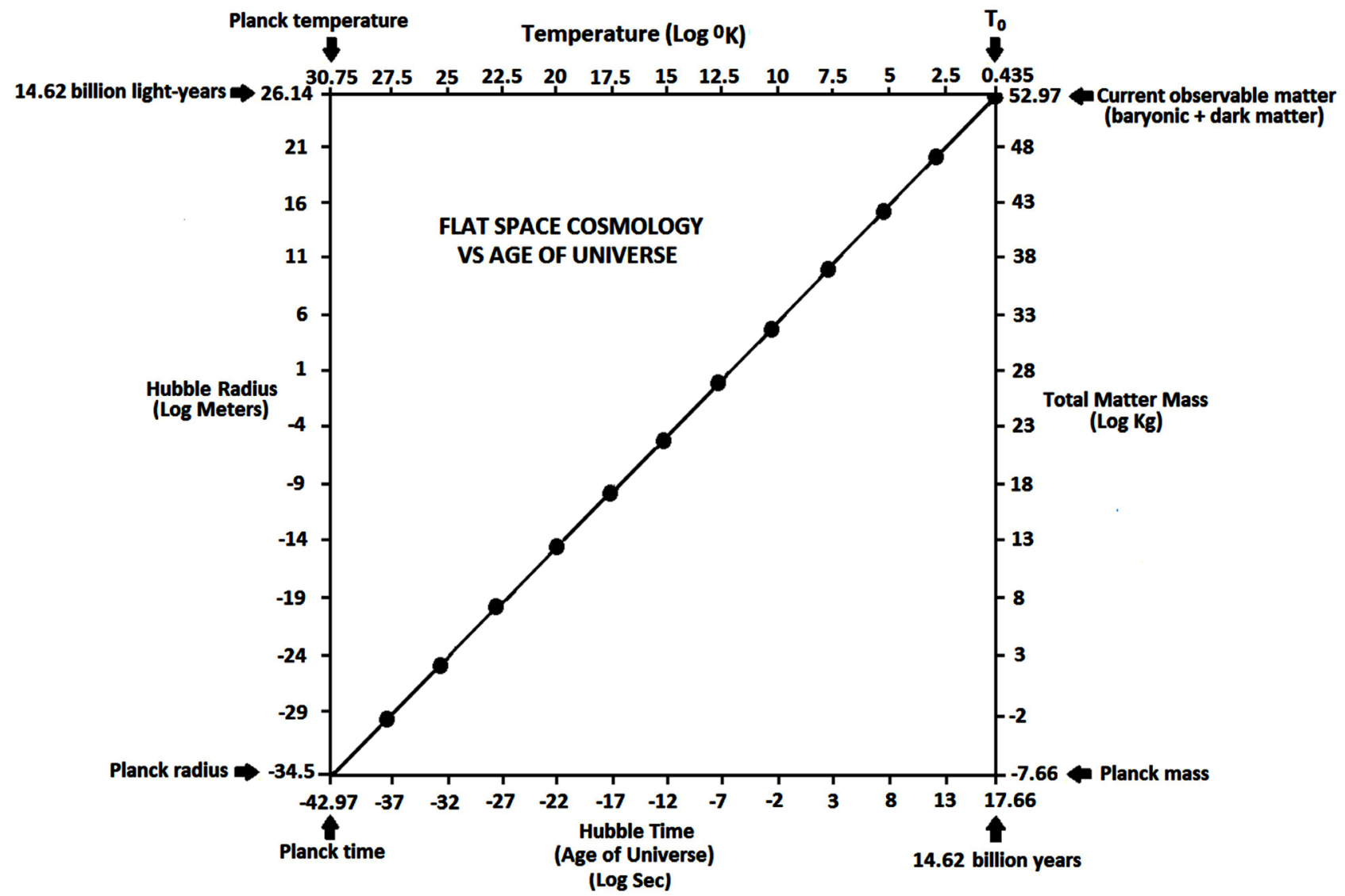

Figure 1. Flat space cosmology parameters as a function of cosmic time. 
derived from the FSC assumptions:

$$
\begin{gathered}
T^{2} R=1.027246639815497 \times 10^{27} \mathrm{~K}^{2} \cdot \mathrm{m} \\
T^{2} t_{s}=3.426525959553982 \times 10^{18} \mathrm{~K}^{2} \cdot \mathrm{s} \\
T^{2} t_{y s}=1.085781647371578 \times 10^{11} \mathrm{~K}^{2} \cdot \mathrm{yr}(\text { sidereal years }) \\
T^{2} t_{y j}=1.085781646054745 \times 10^{11} \mathrm{~K}^{2} \cdot \mathrm{yr}(\text { Julian years })
\end{gathered}
$$

In defining a new cosmological temperature scale ("Universal Temperature" $T_{u}$ ), by $T_{u}=T^{2}$, the Kelvin temperature scale can be converted to a finer scale which also scales by roughly $60.63 \operatorname{logs}$ of 10 . Therefore, the $T^{2} R$ and $T^{2} t$ formulae above assume the new form of $T_{u} R$ and $T_{u} t$ of the same numerical values. Graphs of $T_{u}$ as a function of time, radius or total matter mass then become perfectly symmetrical about the $x=y\left(i . e ., T_{u}=t, T_{u}=R, T_{u}=M\right)$ axis, with the vertical and horizontal axes acting as asymptotes. These graphs are shown below in Figure 2, Figure 3 and Figure 4, respectively.

Thus, the FSC cosmic model can be shown to be finite at any point in time, but unbounded in terms of a beginning time or ending time. And, by incorporating

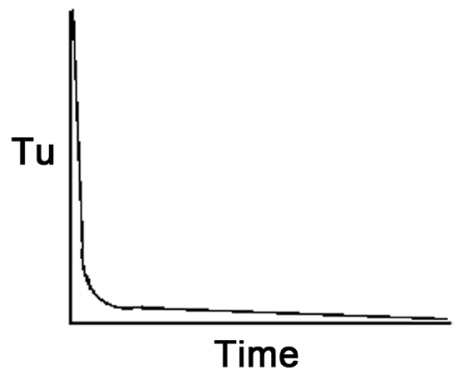

Figure 2. Universal Temp vs Time.

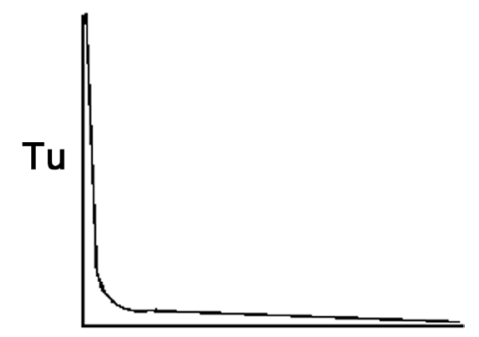

Radius

Figure 3. Universal Temp vs Radius.

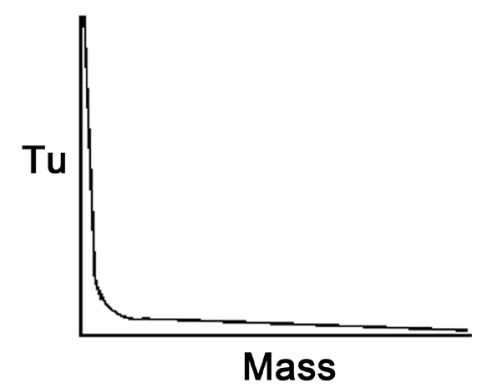

Figure 4. Universal Temp vs Mass. 
the above new FSC definition of "Universal Temperature" (i.e., $T_{u}=T^{2}$ ), a new FSC log graph can be presented in Figure 5 below.

While there is complete one-to-one correspondence between the new $T_{u}$ scale and the $T$ (Kelvin) scale, the symmetry of scaling with $T_{u}$ is considered to be an attractive feature of this model. This new temperature scaling method will be used in future FSC papers.

\section{Kelvin Temperature Scaling in the FSC vs Standard Model}

As first reported in the referenced FSC thermal radiation redshift paper [Tatum (2015)], redshift $\mathrm{z}$ is related to cosmic temperature by

$$
z \cong\left(\frac{T_{t}^{2}}{T_{o}^{2}}-1\right)^{1 / 2}
$$

In standard cosmology, the following formula is used

$$
T_{C M B} \cong 2.725(1+z)
$$

where $T_{C M B}$ represents the cosmic microwave background radiation temperature [24]. These temperature vs redshift formulae give temperature curves which reflect the basic differences between FSC and standard cosmology. These show very subtle differences at low non-zero $\mathrm{z}$ values, but potentially important differences at high $\mathrm{z}$ values. For instance, the temperature differences between these two models may be important with respect to the timing of the "cosmic dawn"

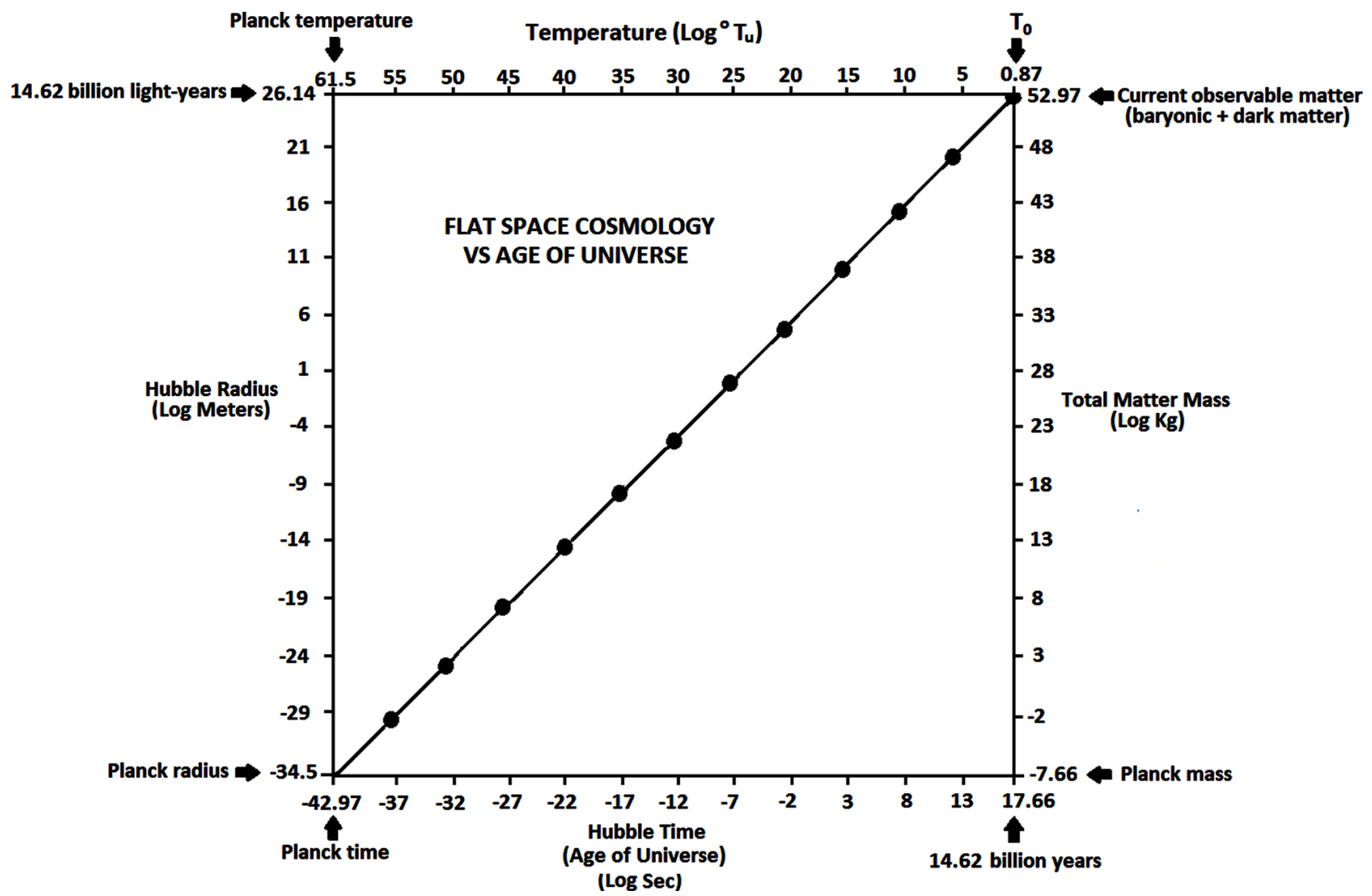

Figure 5. Flat space cosmology parameters as a function of cosmic time. 


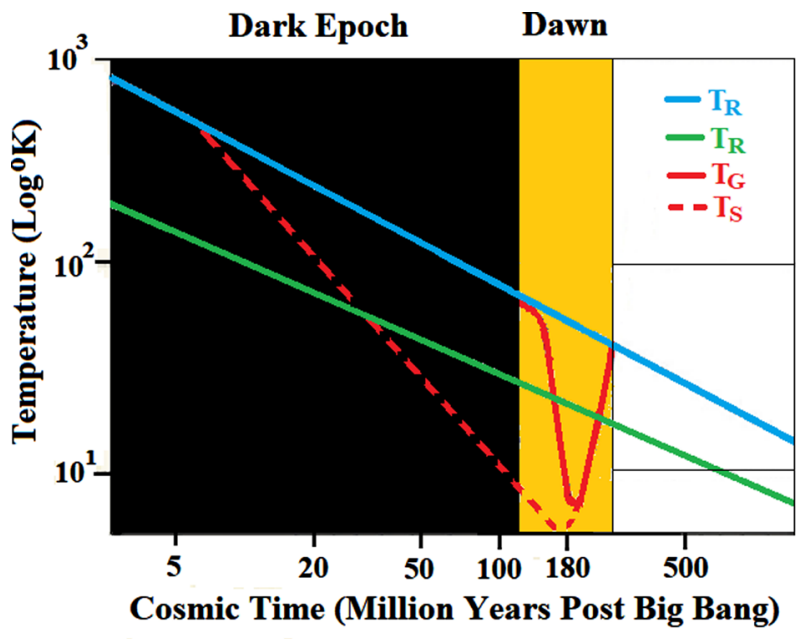

Figure 6. Kelvin temperature vs cosmic time in standard (blue) and FSC (green) models.

epoch emerging from the "dark age" epoch. Figure 6 shows how these models differ with respect to this transition period as a function of cosmic years after the Big Bang [25].

The $T_{R}, T_{G}$ and $T_{\mathrm{S}}$ symbols stand for $\mathrm{CMB}$ radiation temperature, baryonic gas temperature and spin temperature, respectively. The blue line is the standard model temperature curve and the green line is the FSC temperature curve. The precipitous gas temperature dip is presumed to be triggered by temperature-induced interaction with (colder) dark matter, and it is proposed that this event can be measured by $21 \mathrm{~cm}$ radiation redshifted at about $z=20$. This proposed beginning event of cosmic dawn is also proposed as ending when the $T_{\mathcal{S}}$ and $T_{G}$ values rise back up to $T_{R}$ at a redshift of approximately $Z=15$. What is notable about the FSC $T_{R}$ curve in comparison to the standard model $T_{R}$ curve is that the FSC model cosmic dawn would start as early as about 20 million years after the Big Bang, as opposed to the proposed beginning of cosmic dawn at about 110 million years after the Big Bang according to the standard model.

If one wishes to compare both models to an even earlier event in cosmic time, consider the "recombination epoch" event which emitted the CMB radiation and is supposed to have happened at the cosmic temperature of $3000 \mathrm{~K}$. The standard model indicates this event to have occurred approximately 380,000 years after the Big Bang, while the FSC model, by Equation (12), indicates this event to have occurred approximately 12,064 years after the Big Bang. Here, of course, one is assuming an operational definition that the Big Bang occurred at the Planck scale. Obviously, an unbounded FSC model does not single out the Planck scale as a "beginning" any more than any other smaller or larger scale.

\section{Summary and Conclusions}

Temperature scaling is redefined in this paper in terms of a new "Universal Temperature" $T_{u}$ scale according to $T_{u}=T^{2}$, where $T^{2}$ is in $\mathrm{K}^{2}$. This rescaling puts FSC cosmic temperature, time, total matter mass, and Hubble radius on the 
same scale, covering roughly 60.63 logs of 10 from the Planck scale to the present scale.

Even the larger differences in cosmic temperature vs cosmic time, in the early universe, are fairly small between the FSC and standard cosmological models. However, it is becoming apparent that the farther back in time we look, the more we are surprised to see how early the first stars and galaxies formed. Since new cosmic epochs begin when the falling cosmic temperature reaches some threshold value, the most likely explanation for these recent observational surprises is that the standard model temperature curve as a function of cosmic time is slightly higher than the actual curve. This paper suggests that, because the FSC model temperature/time curve is lower at each stage of cosmic time, FSC may actually be a better fit for the timing of these early cosmic events.

Further observational studies of the behavior of dark matter, particularly with respect to $21 \mathrm{~cm}$ scattering interactions with baryonic gas particles [Barkana (2018)], may ultimately prove the superiority of the FSC dynamic dark energy model with respect to the standard cosmology model [26].

\section{Dedications and Acknowledgements}

Both authors dedicate this paper to Dr. Stephen Hawking and Dr. Roger Penrose for their ground-breaking work on black holes and their possible application to cosmology. Dr. Tatum thanks Dr. Rudolph Schild of the Harvard Center for Astrophysics for his past support and encouragement. Author Seshavatharam UVS is indebted to professors Brahmashri M. NagaphaniSarma, Chairman, Shri K.V. Krishna Murthy, founding Chairman, Institute of Scientific Research in Vedas (I-SERVE), Hyderabad, India, and to Shri K.V.R.S. Murthy, former scientist IICT (CSIR), Govt. of India, Director, Research and Development, I-SERVE, for their valuable guidance and great support in developing this subject.

\section{References}

[1] Penrose, R. (1965) Physical Review Letters, 14, 57. https://doi.org/10.1103/PhysRevLett.14.57

[2] Hawking, S. and Penrose, R. (1970) Proceedings of the Royal Society of London A, 314, 529-548. https://doi.org/10.1098/rspa.1970.0021

[3] Tatum, E.T., Seshavatharam, U.V.S. and Lakshminarayana, S. (2015) International Journal of Astronomy and Astrophysics, 5, 116-124. https://doi.org/10.4236/ijaa.2015.52015

[4] Tatum, E.T., Seshavatharam, U.V.S. and Lakshminarayana, S. (2015) Journal of Applied Physical Science International, 4, 18-26.

[5] Tatum, E.T., Seshavatharam, U.V.S. and Lakshminarayana, S. (2015) Frontiers of Astronomy, Astrophysics and Cosmology, 1, 98-104.

[6] Planck Collaboration XIII (2016) Astronomy \& Astrophysics, 594, A13. http://arxiv.org/abs/1502.01589

[7] Dicke, R.H. (1970) Gravitation and the Universe. American Philosophical Society.

[8] Guth, A.H. (1997) The Inflationary Universe. Basic Books, New York. 
[9] Guth, A.H. (1981) Physical Review D, 23, 347. https://doi.org/10.1103/PhysRevD.23.347

[10] Albrecht, A. and Steinhardt, P.J. (1982) Physical Review Letters, 48, 1220-1223. https://doi.org/10.1103/PhysRevLett.48.1220

[11] Linde, A.D. (1982) Physics Letters, 108B, 389-392. https://doi.org/10.1016/0370-2693(82)91219-9

[12] Linde, A. (1990) Inflation and Quantum Cosmology. Academic Press, Boston. https://doi.org/10.1017/CBO9780511564178.016

[13] Perlmutter, S., et al. (1999) Astrophysical Journal, 517, 565-586. https://doi.org/10.1086/307221

[14] Schmidt, B., et al. (1998) Astrophysical Journal, 507, 46-63. https://doi.org/10.1086/306308

[15] Riess, A.G., et al. (1998) Astronomical Journal, 116, 1009-1038. https://doi.org/10.1086/300499

[16] Tutusaus, I., et al. (2017) Astronomy \& Astrophysics, 602, A73.

[17] Dam, L.H., et al. (2017) Monthly Notices of the Royal Astronomical Society, 472, 835-851. https://doi.org/10.1093/mnras/stx1858

[18] Nielsen, J.T., et al. (2015) Scientific Reports, 6, Article No. 35596. https://doi.org/10.1038/srep35596

[19] Wei, J.-J., et al. (2015) Astronomical Journal, 149, 102-112. https://doi.org/10.1088/0004-6256/149/3/102

[20] Melia, F. (2012) Astronomical Journal, 144, 110.

[21] Bekenstein, J.D. (1974) Physical Review D, 9, 3292-3300. https://doi.org/10.1103/PhysRevD.9.3292

[22] Hawking, S. (1976) Physical Review D, 13, 191-197. https://doi.org/10.1103/PhysRevD.13.191

[23] Inoue, A.K. (2004) Monthly Notices of the Royal Astronomical Society, 350, 729-744. https://doi.org/10.1111/j.1365-2966.2004.07686.x

[24] Barkana, R. (2018) Cosmic Dawn as a Dark Matter Detector.

[25] Bowman, J.D. (2018) Nature, 555, 67-70. https://doi.org/10.1038/nature25792

[26] Zhao, G., et al. (2017) Nature Astronomy, 1, 627-632. 